\title{
Avaliação da resistência mecânica e absorção de água em cerâmica vermelha com incorporação de chamote
}

Evaluation of mechanical resistance and water absorption in heavy clay ceramic with brick waste incorporation

\author{
A. Zaccaron ${ }^{1 *}$; S. L. Galatto ${ }^{1,2}$; V. S. Nandi ${ }^{3}$; P. Fernandes ${ }^{3}$ \\ ${ }^{1}$ Departamento de Engenharia Ambiental, Universidade do Extremo Sul Catarinense - UNESC, 88806-000, Criciúma- \\ SC, Brasil \\ ${ }^{2}$ Instituto de Pesquisas Ambientais e Tecnológicas - IPAT, Universidade do Extremo Sul Catarinense - UNESC, 88806- \\ 000, Criciúma-SC, Brasil \\ ${ }^{3}$ Departamento de Engenharia Cerâmica, Centro Universitário Barriga Verde - UNIBAVE, 88845-000, Cocal do Sul- \\ SC, Brasil
}

*alexandrezaccaron@hotmail.com

(Recebido em 19 de março de 2017; aceito em 04 de fevereiro de 2018)

\begin{abstract}
O presente estudo visa analisar as propriedades tecnológicas de resistência mecânica e absorção de água em peças de cerâmica vermelha, com adição de diferentes percentuais de resíduos proveniente do processo pósqueima da fabricação de blocos de vedação (chamote). O estudo foi realizado em duas etapas, sendo a primeira a avaliação da massa padrão (STD) para produção de cerâmica vermelha e outras quatro formulações com diferentes percentuais $(5,10,15$ e $20 \%)$ de adição do chamote como matéria-prima alternativa. Nessa primeira etapa, os corpos-de-prova foram confeccionados manualmente com auxílio de um molde de PVC. Para a segunda etapa, foram escolhidas a massa padrão (STD) e a formulação com $20 \%$ para serem conformadas por extrusão e avaliar as propriedades tecnológicas em questão conforme a norma técnica ABNT NBR 15270. As peças foram queimadas - em ambas as etapas - a $900{ }^{\circ} \mathrm{C}$, simulando os procedimentos padrão das indústrias cerâmicas. Foi possível observar na primeira etapa, que a resistência mecânica dos corpos-de-prova tende a diminuir com o aumento da absorção de água, como consequiência da adição de maiores percentuais de chamote. Todavia, essa diferença se dá de forma pouco relevante. Já na segunda etapa, apesar da diferença ocasionada pela adição do resíduo, os corpos-de-prova atenderam as normas, ao serem ensaiados nas propriedades estudadas.
\end{abstract}

Palavras-chave: cerâmica vermelha, chamote, resíduo

This study aims to analyze the technological properties of mechanical strength and water absorption in heavy clay ceramic, with the addition of different levels of residues from fired bricks (brick powder). The study was carried out in two stages, the first being the standard mass (STD) for the production of heavy clay ceramics and four other formulations with different percentages $(5,10,15$ and $20 \%)$ of the addition of brick powder waste as an alternative raw material. In this first step, the samples were manually made using a PVC mold. For the second step, the standard mass (STD) and formulation with $20 \%$ were chosen to be extrusion formed and to evaluate the technological properties in question according to the Brazilian technical standard ABNT NBR 15270. The pieces were burned - in both steps - at $900^{\circ} \mathrm{C}$, simulating the ceramic industries procedures. It was possible to observe in the first stage that the mechanical strength of the sample tends to decrease with the increase of the water absorption, as a consequence of the addition of greater percentages of brick powder waste. However, this difference is insignificant. In the second stage, despite the difference caused by the addition of the waste, the samples met the standards, when they were tested in the studied properties.

Keywords: heavy clay ceramic, brick powder, waste

\section{INTRODUÇÃO}

A cerâmica está presente em diferentes civilizações há milhares de anos, sendo um dos primeiros materiais usados pelo homem, como artesanato na fabricação de potes e jarros, e posteriormente como implementos na construção. A história do tijolo pode ser contada como simultâneo à evolução do homem. Desde o adobe moldado até a confecção de blocos queimados com boa rigidez, ultrapassando o tempo até chegar ao cenário atual de extrema evolução fabril [1-9] 
A produção de elementos cerâmicos, como tijolos, blocos e telhas, terá uma procura ascendente nos próximos anos, principalmente nos países em desenvolvimento, o que necessitará de maiores volumes de matéria-prima bruta (argila) e consequentemente, aumentará a geração de resíduos sólidos provenientes do processo pós-queima [10-11].

O beneficiamento de matérias-primas e seus produtos geram resíduos que muitas vezes são agressivos ao meio ambiente. A destinação adequada desses rejeitos deve ser objetivo constante do setor produtivo. Uma das alternativas é o seu reaproveitamento através da reciclagem ou adição em processos produtivos [13-14].

O Brasil produz cerca de 130 milhões de toneladas de cerâmica vermelha anualmente, algo em torno de 5,3 bilhões de peças/mês, com uma demanda de material argiloso que chega na casa das 10,3 milhões ton/mês [15]. Diante desses números de produção, é importante destacar que os resíduos industriais constituem-se atualmente um dos principais problemas ambientais a serem enfrentados pelo setor industrial, que encontra grandes dificuldades em gerenciá-los de modo adequado. Na cerâmica vermelha estima-se uma perda entre 3 e $5 \%$ de sua produção total, percentual formado de produtos cerâmicos quebrados e com algum defeito no pós-queima que não são comercializados. É importante ressaltar que a maior parte dos resíduos gerados no processo pós queima não são reaproveitados, tornando-se empecilho para manuseio, além de custos com sua deposição final em aterro [16-17].

O chamote pode ser definido como um subproduto proveniente dos rejeitos de material cerâmico após a queima, no qual deve ser beneficiado após o seu descarte, procedimento geralmente realizado por moinho de martelos, no qual visa chegar a um nível de fragmentação aceitável a fim de ser empregado no processo fabril [18-19].

De acordo com a caracterização e classificação do resíduo, efetuada segundo a ABNT/NBR 10004:2004 [20], o chamote oriundo da região do extremo sul catarinense apresenta concentrações de manganês acima do permitido pela norma, sendo classificado como resíduo não perigoso, porém não inerte (Classe II A - não inerte, fazendo com que haja a necessidade de deposição em ambiente controlado) [21].

Esse material vem sendo assunto de pesquisas no mundo todo, visando sua aplicação no processo fabril, já que na produção ele é considerado inerte até sua temperatura de obtenção, e apresenta algumas propriedades tecnológicas, tais como a retração de secagem e principalmente como agente desplastificante (material não-plástico) que auxilia na secagem, evitando trincas e outros problemas enfrentados durante o tratamento térmico de secagem [18, 21-30].

$\mathrm{O}$ presente estudo tende a analisar a influência da adição do chamote nas propriedades tecnológicas de absorção de água e resistência mecânica, que são geridas pela norma técnica ABNT/NBR 15270:2005 [31-32] e tem importância fundamental na qualidade final das peças cerâmicas.

\section{MATERIAL E MÉTODOS}

Para a realização do estudo, foi utilizada uma massa padrão de uma indústria cerâmica sulcatarinense que disponibilizou sua matéria-prima, que é composta por uma mistura de duas argilas, no qual foram apanhadas diretamente no Box de armazenamento de argilas. Foram coletados aproximadamente $100 \mathrm{~kg}$ de uma argila plástica e $100 \mathrm{~kg}$ de uma argila arenosa, ambas de 5 pontos diferentes. Posteriormente as amostras passaram pelo processo de quarteamento, homogeneizadas e reduzidas para um volume de aproximadamente $50 \mathrm{~kg}$ de cada uma das argilas. O chamote foi cedido pela empresa, já desagregado e beneficiado, em uma granulometria apropriada para ensaios laboratoriais.

Já no laboratório, as argilas e o chamote foram desidratados em estufa (De Leo, 2211, 8) a 100 $\pm 5{ }^{\circ} \mathrm{C}$. As argilas passaram por desagregação por laminação (Laminador BERTAN), para blenda a base seca. Os ensaios foram realizados em duas etapas. Na primeira foi realizada a conformação manual dos corpos-de-prova, cujas formulações realizadas encontram-se na Tabela 1, e apresentados no gráfico ternário da Figura 1. 
Tabela 1: Composições da massa cerâmica com resíduo incorporado

\begin{tabular}{lccccc}
\hline \multicolumn{1}{c}{ Matérias- } & \multicolumn{5}{c}{ Formulação (\%massa) } \\
\cline { 2 - 6 } \multicolumn{1}{c}{ primas } & STD & $\mathbf{5}$ & $\mathbf{1 0}$ & $\mathbf{1 5}$ & $\mathbf{2 0}$ \\
\hline $\begin{array}{l}\text { Argila } \\
\text { plástica }\end{array}$ & 60 & 57,5 & 55 & 52,5 & 50 \\
$\begin{array}{l}\text { Argila } \\
\text { arenosa }\end{array}$ & 40 & 37,5 & 35 & 32,5 & 30 \\
Chamote & 0 & 5 & 10 & 15 & 20 \\
\hline TOTAL & 100 & 100 & 100 & 100 & 100 \\
\hline
\end{tabular}

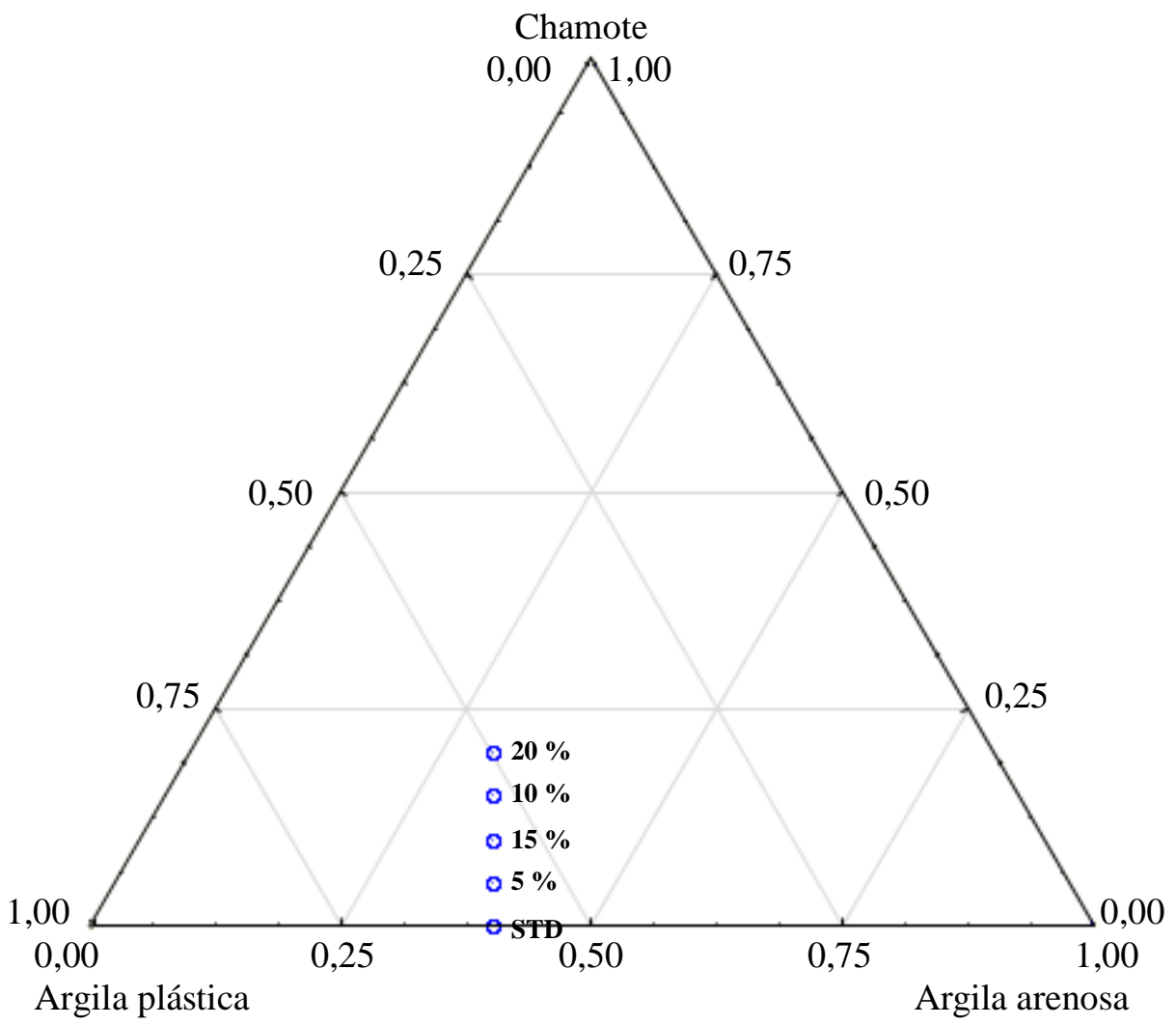

Figura 1: Diagrama ternário apontando as formulações desenvolvidas

A etapa 2 consistiu na confecção de corpos-de-prova por extrusão das formulações STD e uma segunda com $20 \%$ de chamote incorporado.

Para a etapa 1 (conformação manual), as formulações foram umidificadas uniformemente e postas para descansar por $24 \mathrm{~h}$ para uma melhor homogeneização da umidade. Após esse período, foram confeccionados os corpos-de-prova, com auxílio de um molde de PVC com diâmetro aproximado de $35 \mathrm{~mm}$ e $16 \mathrm{~mm}$ de altura. As peças ficaram em repouso para secagem natural por $24 \mathrm{~h}$, e posteriormente foram postas em secagem forçada em estufa (DeLeo, 2211, 8) a uma temperatura de $100 \pm 10{ }^{\circ} \mathrm{C}$ por mais $24 \mathrm{~h}$. Após a etapa de secagem, as amostras foram levadas para queima em forno laboratorial tipo mufla (Jung, Modelo J200), com taxa de aquecimento de 2 ${ }^{\circ} \mathrm{C} / \mathrm{min}$, temperatura de queima de $900{ }^{\circ} \mathrm{C}$ e tempo de patamar de 2 horas.

Uma vez queimadas, os corpos-de-prova da etapa 1 passaram pelo ensaio de absorção de água conforme norma técnica ABNT/NBR 15270:2005 [32] e resistência mecânica diametral, em uma máquina universal de ensaios (EMIC DL-20000). Foi realizada a observação da porosidade dos corpos-de-prova da etapa 1 (STD e $20 \%$ ), com auxílio de um Microscópio BX41M-LED da marca Olympus.

$\mathrm{Na}$ Etapa 2, as massas foram laminadas (laminador BERTAN) e passaram pelo processo de extrusão (extrusora laboratorial da marca NATREB), com uma pressão de vácuo que variou entre 
0,5 a 0,84 atm. O tratamento térmico dessa etapa foi sob as mesmas condições da etapa 1 . As propriedades tecnológicas observadas foram a absorção de água e resistência mecânica à compressão em uma máquina universal de ensaios (EMIC DL-20000), ambos os testes realizados conforme norma técnica ABNT/NBR 15270:2005 [32].

\section{RESULTADOS E DISCUSSÃO}

Uma das particularidades da cerâmica para alvenaria são os valores de porosidade. Segundo a literatura [33], quando verde, a mesma depende somente das frações granulométricas dos componentes e do modo com o qual estão misturados. Uma melhor compactação e um número reduzido de poros são obtidos através de uma distribuição perfeita de granulometria, com grânulos pequenos que preenchem os espaços deixados pelos maiores. Após o processo de queima, a porosidade tende a aumentar devido à eliminação de matéria orgânica e da água.

A Figura 2 ilustra a microscopia ótica dos corpos-de-prova sem nenhum percentual de resíduo incorporado - STD (A) e com $20 \%$ de resíduo incorporado (B).
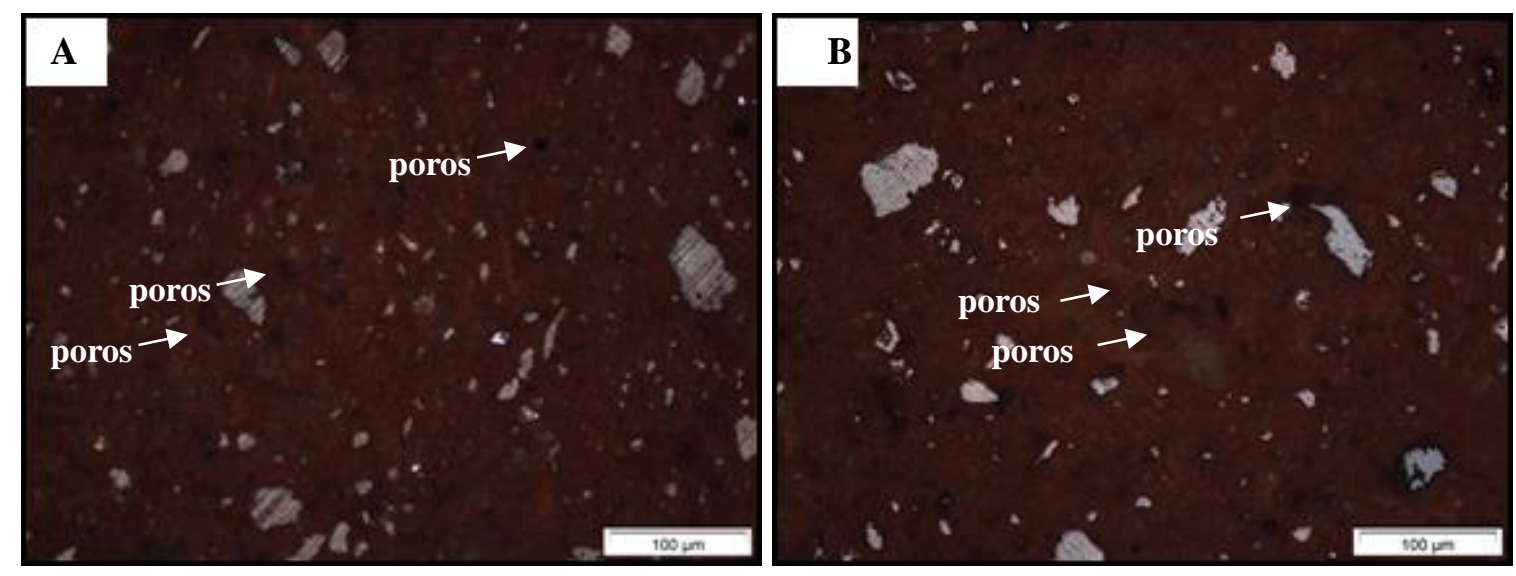

Figura 2: Microscopia para observação da porosidade das peças (A) STD e (B) $20 \%$.

É possível observar que há uma grande incidência de poros nos corpo-de-prova cerâmicos, e essa ocorrência tende a crescer com maiores teores de resíduo incorporado, muito devido ao fato das granulometrias não estarem em perfeita simetria, fazendo com que não haja preenchimento de toda a área, ocasionando os poros.

É importante destacar, que para fabricação de cerâmica para alvenaria, é fundamental o controle da porosidade na peça, uma vez que esta está diretamente ligada com outras propriedades qualitativas dos produtos cerâmicos. A absorção de água, por exemplo, está diretamente associada à quantidade de poros da peça, uma vez que, ao entrar em contato com a umidade, a água ocupa os espaços vazios na peça, preenchendo-os totalmente, e estabelecendo aí, a absorção de água.

Outro fator de extrema relevância está ligado entre absorção de água e resistência mecânica, uma vez que a peça cerâmica tem seu pior desempenho em contato com a água [21].

A Figura 3 apresenta o gráfico com o percentual de absorção de água versus resistência mecânica diametral, realizados na etapa 1 . É possível observar uma leve tendência da absorção de água aumentar com maiores teores de resíduo incorporado, um acréscimo de aproximadamente $2 \%$. Mesmo com o aumento de absorção de água, o ensaio de resistência mecânica diametral se manteve praticamente o mesmo, independente do valor de resíduo incorporado. 


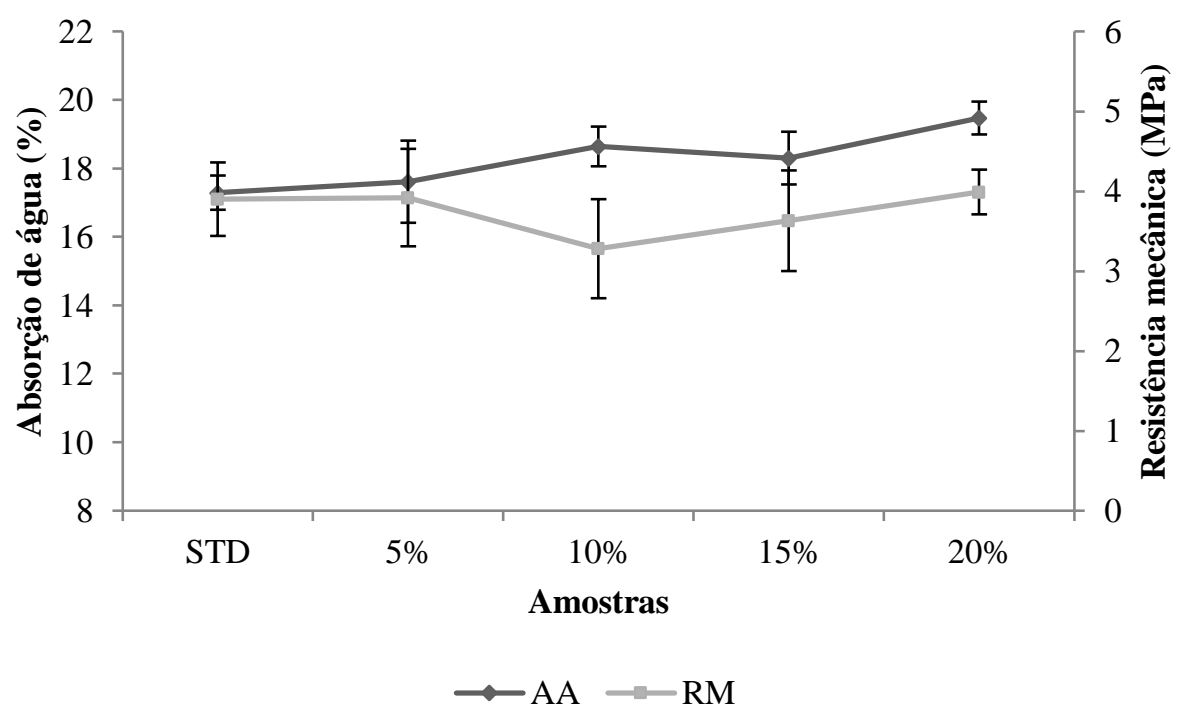

Figura 3: Gráfico com o percentual de absorção de água versus resistência mecânica diametral

Os mesmos ensaios foram realizados com as peças confeccionadas na Etapa 2, que são apresentados nos gráficos das figuras 4 e 5 . Devido à utilização do processo de extrusão à vácuo no processamento se obtém corpos de provas com menor porosidade e melhor homogeneidade, densificando e unificando a massa utilizada para o processo de conformação. Ou seja, há um menor índice de variação entre os corpos-de-prova analisados e maior precisão do resultado. Pode-se observar na figura 4 que o chamote não cumpriu sua função de preencher os espaços vazios e que serviu como ponte de acesso para ligar a água aos poros existentes. Uma vez sabendo que o chamote também tem capacidade de absorver água, nota-se um aumento significativo em tal propriedade nas provas com $20 \%$ de chamote agregado. Apesar disso, o aumento de aproximadamente $3 \%$ não ultrapassa os limites estabelecidos por norma [31] que dita os limites mínimos e máximos sendo de 8 a $22 \%$.

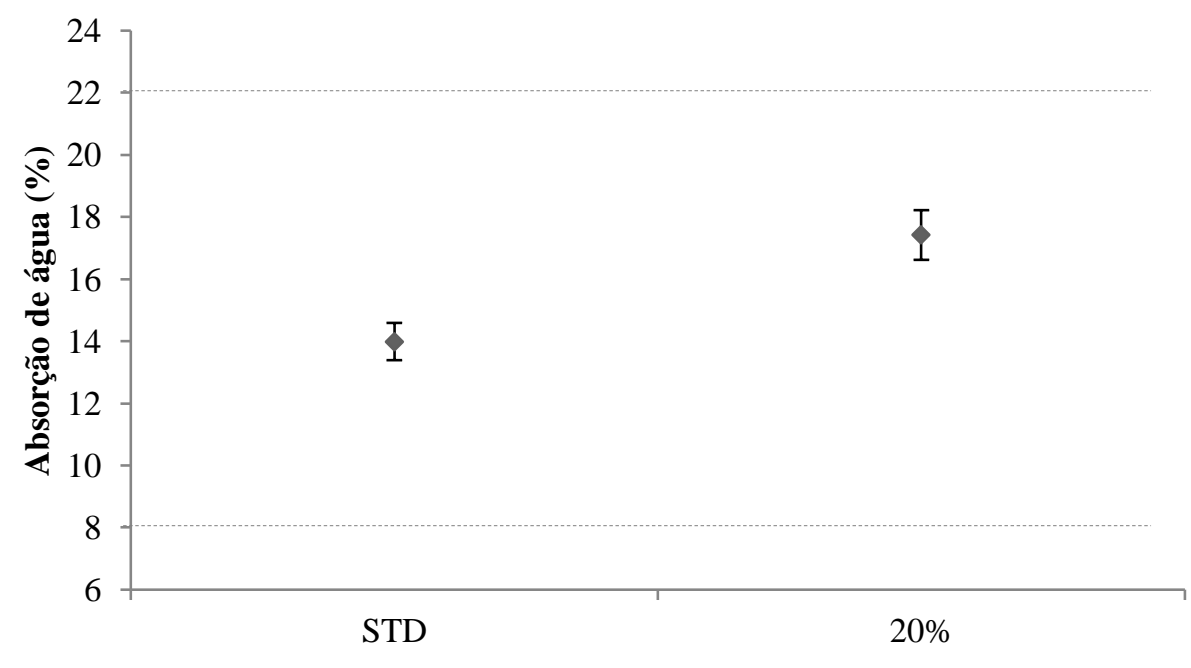

Amostras

Figura 4: Gráfico com o percentual de absorção de água da etapa 2

Para resistência mecânica, devido à baixa granulometria do chamote, na temperatura utilizada apresentou uma leve fundência, a ponto de envolver com formação de fase liquida as argilas e os minerais presentes, ocorrendo em uma maior solidificação, o que resultou em um leve aumento da resistência mecânica como apresentado na figura 5. A densificação do material 
cerâmico por sua vez não ocorreu a ponto de bloquear a passagem de água em sua formação pós queimada, apresentando capacidade de absorver água a medida que se agrega maiores percentuais de chamote. É importante destacar que, para esse ensaio, os resultados foram comparados com a norma vigente [31], onde as peças devem atender resistência mínima de 1,5 MPa, e em ambos os casos, ficaram dentro da diretriz da referida normativa.

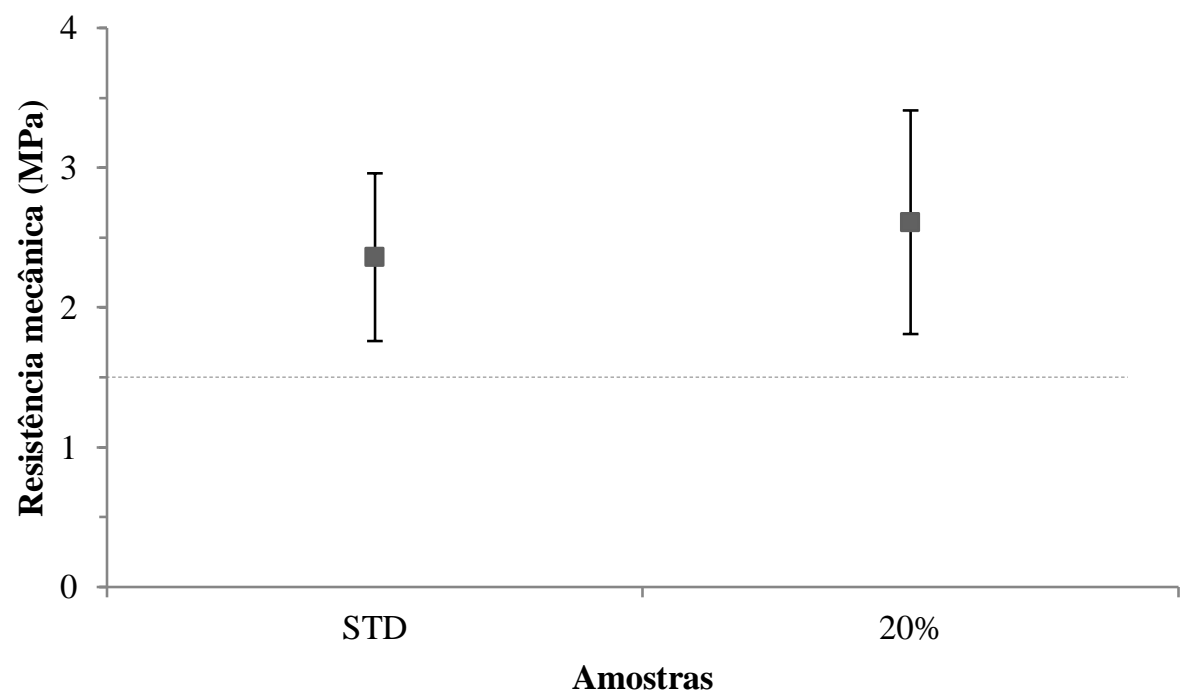

Figura 5: Gráfico com a resistência mecânica da etapa 2

\section{CONCLUSÃO}

Os ensaios realizados nos corpos-de-prova que simulam as condições fabris, mostraram que a incorporação em até $20 \%$ de chamote na massa cerâmica para fabricação de blocos de vedação atende as normas técnicas.

A adição do chamote aumentou a porosidade na peça, o que ocasionou o aumento da absorção de umidade, porém ficando dentro da norma técnica. Para o teste de resistência mecânica, a incorporação do chamote não apresentou influência, onde os resultados se mantiveram praticamente inalterados, independentemente do percentual adicionado.

Se o Brasil consome aproximadamente 130 milhões de toneladas de argila todos os anos para a fabricação de cerâmica vermelha e estima-se uma perda de 3 a $5 \%$ de produtos pós-queima, o país tem um desperdício anual de aproximadamente 5 milhões de toneladas de resíduos de quebra, que se incorporados no processo, resultariam na diminuição do consumo de argila bruta.

\section{AGRADECIMENTOS}

Os autores agradecem ao CNPq, a CAPES e a todas as empresas que colaboraram direta ou indiretamente para o desenvolvimento deste trabalho.

\section{REFERÊNCIAS BIBLIOGRÁFICAS}

1. Perez-Monserrat EM, Agua F, Fort R, de Buergo MA, Conde JF, García-Heras M. Effect of manufacturing methods on the decay of ceramic materials: A case study of bricks in modern architecture of Madrid (Spain). Applied Clay Science, 2017 Jan;135:136-149, doi:10.1016/j.clay.2016.09.015

2. Ukwatta A, Mohajerani A, Setunge S, Eshtiaghi N. Possible use of biosolids in fired-clay bricks, Construction and Building Materials, 2015 Aug;91:86-93, doi:10.1016/j.conbuildmat.2015.05.033

3. Lima CC. A matéria como significado: a relação entre técnica cerâmica e mitologia em diferentes culturas. In: Congresso Nacional das Artes do Fogo, 2012, São Paulo. Contaf 2012 - Congresso Nacional para técnicas das Artes do Fogo. São Paulo, SP, 2012. 
4. Deboucha S, Hashim R. A review on bricks and stabilized compressed earth blocks. Scientific Research and Essays, 2011 Feb;6(3):499-506, doi:10.5897/SRE09.356.

5. Pacheco Torgal F, Jalali S. Eco-efficient Construction and Building Materials. London: Springer London, 2011, 247p. doi:10.1007/978-0-85729-892-8.

6. Alvarez JAS. Alvenarias e Argamassas anteriores ao Império Romano. In: APFAC, $2^{\circ}$ Congresso Nacional de Argamassas de Construção, Lisboa. 2007.

7. Kornmann M. Clay Bricks and Roof Tiles: Manufacturing and Properties. Ed.: Societé de l'Industrie Minerale, Paris, 2007, 308 p.

8. Yasui I. Ceramics, History of. Encyclopedia of Condensed Matter Physics. [S.1.]: Elsevier, 2005, p. 173177.

9. Campbell JWP., Pryce W. Brick: A World History, Publisher: Thames \& Hudson, First Edition, 2003, $320 \mathrm{p}$.

10. Amin SK, Sibak HA, El-Sherbiny SA, Abadir MF. An overview of ceramic wastes management in construction. International Journal of Applied Engineering Research, 2016;11(4):2680-2685.

11. Zhang L. Production of bricks from waste materials - A review, Construction and Building Materials, 2013 Oct;47:643-655, doi:10.1016/j.conbuildmat.2013.05.043.

12. Quintana LMH. Avaliação das matérias-primas e produtos cerâmicos da região de Bagé - RS. [Dissertação]. Santa Maria (RS): CPGEC/UFSM; 2000. 116 p.

13. Quintana LMH, Soares JMD. The use of Rice husk ash in the production of structural ceramics In: Symposium on Construction \& Environment: Theory Into Pratice - CIB2000, USP, São Paulo (SP), 2000, 9 p.

14. Silva NIW, Zwonok O, Chies F. Artefatos cerâmicos obtidos a partir de misturas entre argilas e cinzas de carvão, In: $41^{\circ}$ Congresso Brasileiro de Cerâmica, São Paulo(SP), 1997, p.285-288.

15. ANICER[Associação Nacional da Indústria Cerâmica] Dados Oficiais, 2008. Disponível na internet em: http://www.anicer.com.br/index.asp?pg=institucional.asp\&secao=3\&categoria=60\&selMenu=3 Acesso em 08/04/2015.

16. Vasconcelos F. Restos de tijolos formam bloco mais resistente, SECOM - Universidade de Brasília, 2009, Disponível na Internet em: http://www.arqbrasil.arq.br/_arqdoc/textos/arqdoc_027.htm Acesso em 08/04/2015.

17. IPT [Instituto de Pesquisas Tecnológicas]. Aplicação de cacos cerâmicos: Estudo do IPT analisa formas de uso dos resíduos de cerâmica vermelha, visando reduzir passivos ambientais. 2014. Disponível na internet em: www.ipt.br/noticia/780. Acesso em 08/04/2015.

18. Vieira CMF, Teixeira SS, Monteiro SN. Efeito da Temperatura de queima nas propriedades e microestrutura de cerâmica vermelha contendo chamote. Cerâmica, 2009 Jul/Sep; 55(335):332-336, doi:10.1590/S0366-69132009000300014

19. Dondi M, Fabri B, Marsigli M. Resenha das experiências de reciclagem de resíduos industriais e urbanos na produção de tijolos. Cerâmica Informação, 1998;1:17-29.

20. ABNT [Associação Brasileira de Normas Técnicas] NBR 10004/2004. Resíduos Sólidos - Classificação de Resíduos Sólidos: Rio de Janeiro: ABNT, 2004, 71p.

21. Zaccaron A, Galatto SL, Nandi VS, Fernandes P. Incorporação de chamote na massa de cerâmica vermelha como valorização de resíduo. Cerâmica Industrial, 2014 May/Jun;19(3): 33-39, doi:10.4322/cerind.2014.077.

22. Oliveira YL, Linhares Júnior Z, Ancelmo L, Soares RAL. Estudo da Reutilização de Resíduos de Telha Cerâmica (Chamote) em Formulação de Massa para Blocos Cerâmicos. Cerâmica Industrial, 2016 Mar/Apr;21(2):45-50, doi:10.4322/cerind.2016.013.

23. Morais MDP, Macedo RS, Raposo CMO. Preparação e caracterização de sistemas chamote/argila visando o reaproveitamento de descartes industriais. Cerâmica, 2015;61(358):206-212, doi:10.1590/036669132015613581864.

24. Rajamannan B, Viruthagiri G, Jawahar KS. Effect of grog addition on the technological properties of ceramic brick. International Journal of Latest Research in Science and Technology, 2013 Nov/Dec;2(6):81-84.

25. Gouveia FP, Sposto RM. Incorporação de chamote em massa cerâmica para a produção de blocos. Um estudo das propriedades físico-mecânicas. Cerâmica, 2009;55(336):415-419, doi:10.1590/S036669132009000400012

26. Gouveia FP, Efeito da incorporação de chamote (resíduo cerâmico queimado) em massas cerâmicas para a fabricação de blocos de vedação para o Distrito Federal-DF. Um estudo experimental. [Dissertação] Universidade de Brasília, Brasília (DF), 2008, 94p.

27. Vieira CMF, Monteiro SN. Effect of grog addition on the properties and microstructure of a red ceramic body for brick production. Construction and Building Materials, 2007 Aug;21(8):1754-1759, doi:10.1016/j.conbuildmat.2006.05.013. 
28. Monteiro SN, Vieira CMF, Carvalho, EA. Technological Behavior of Red Ceramics Incorporated with Brick Waste. Revista Matéria, 2005;10(4):537-542.

29. Vieira CMF, Souza ETA, Monteiro SN. Efeito da incorporação de chamote no processamento e microestrutura de cerâmica vermelha. Cerâmica, 2004 Jul/Sep;50(315):254-260, doi:10.1590/S036669132004000300013

30. Demir I, Orhan M. Reuse of waste bricks in the production line. Building and Environment, 2003 Dec; 38(12):1451-1455, doi:10.1016/S0360-1323(03)00140-9

31. ABNT [Associação Brasileira de Normas Técnicas] NBR 15270/2005. Componentes Cerâmicos - Parte 1: Blocos Cerâmicos para Alvenaria de Vedação - Terminologia e Requisitos: Rio de Janeiro: ABNT, 2005, 11p.

32. ABNT [Associação Brasileira de Normas Técnicas] NBR 15270/2005. Componentes Cerâmicos - Parte 3: Blocos Cerâmicos para Alvenaria Estrutural e de Vedação - Métodos de Ensaio: Rio de Janeiro: ABNT, 2005, 27p.

33. Facincani E. Tecnologia Ceramia i Laterizi. $3^{\text {a }}$ Edizione, Faenza: Gruppo Editoriale Faenza Editrice, 2001, 275 p. 